\title{
COGNITIVE TOOLS FOR ASSESSMENT AND LEARNING IN A HIGH INFORMATION FLOW ENVIRONMENT*
}

\author{
SUSANNE P. LAJOIE, PH.D. \\ McGill University \\ ROGER AZEVEDO, PH.D. \\ Carnegie Mellon University \\ DAVID M. FLEISZER, M.D. \\ McGill University
}

\begin{abstract}
A simulation-based intelligent tutoring system (ITS) was designed for nurses working in a Surgical Intensive Care Unit (SICU). A cognitive task analysis approach was used to identify the cognitive components of clinical decision making of "expert" surgical nurses. Quantitative analyses revealed that expert nurses reached the same decisions. However, a qualitative analysis of the verbal protocols revealed great variability in how the nurses arrived at their clinical decisions. Differences were observed in: hypothesis generation, planning of medical interventions, actions performed, results of evidence gathering, interpretation of the results, heuristics, and the overall solution paths. The results of these analyses were used to design a prototype ITS. The tutoring environment (SICUN) is described in terms of the cognitive tools it provides, and the assessment opportunities it presents. Implications for the evaluation of this system are discussed.
\end{abstract}

\section{RATIONALE}

Research on rapid decision making in real-world domains is still in its infancy. As such, this article attempts to examine the types of decisions nurses make in a high information flow environment, the surgical intensive care unit (SICU). A

*This project is funded by the Quebec Ministry of Industry, Commerce, Science and Technology and partial funds from the Social Science and Humanities Research Council of Canada.

(c) 1998, Baywood Publishing Co., Inc. 
cognitive approach is used to isolate the types of cognitive skills needed to perform in an SICU. These analyses provided the basis for designing a computer based learning environment for medical personnel to foster cognitive proficiency while working in a surgical intensive care unit (SICU). This computer-based learning environment is specifically designed for nurses working in the SICU and hence we have named it SICUN. The SICUN is designed as a problem-based practice environment where nurses practice their patient assessments given a specific patient history. Since this system is still under development, the focus of this article is on describing the methodology used to design the system rather than the evaluation of it.

The design of the SICUN environment is based on cognitive task analysis of problem solving in this domain. The design accommodates individual differences in problem solving by presenting learners with adaptive feedback when a learning impasse occurs. Learners are dynamically assessed in terms of the processes selected for planning and acting within each patient case. The pedagogical strategy is a guided approach to instruction where learners are provided with assistance when requested. Consequently, the assessments in the guided mode can be used to provide adaptive feedback to learners in the context of their problem solving. Computer-based environments, such as the SICUN, are not naturalistic settings, but they are more naturalistic than the traditional experimental paradigms using paper and pencil tasks to extract clinical decision making think alouds. It is possible that these new technologies that approximate cognitive apprenticeship models can provide us with a window on rapid forms of decision making in more realistic settings, and with a much different perspective on decision making than what has been viewed in the past [1].

The theoretical perspective adopted in the design of SICUN is based on the integration of the traditional information processing theory (IPT). Its repertoire of process-tracing methods and more recent advances in the area of naturalistic decision making emphasize situational assessment, mental simulation, and the application of knowledge and expertise in complex, dynamic real world settings. The following section summarizes the theoretical perspectives as they underlie the model of instruction used in SICUN. Subsequent sections of the article address how the computer based learning environment was designed, and how it can be used as a dynamic form of assessment that can guide individual learning experiences.

\section{SITUATIVE LEARNING THEORIES AND MODELS OF INSTRUCTION}

Current learning theories are increasingly centered on the learning process as it occurs within "situations" or meaningful contexts [2-6]. SICUN is designed as a practice environment where nurses can apply their clinical decision making knowledge in realistic problem solving situations. Traditional instructional 
approaches often teach factual knowledge without a mechanism for contextualizing it in situations that would make abstract principles more concrete [7].

From an instructional perspective, the SICU is a natural apprenticeship setting where novices (i.e., nurses new to the ward), learn new content from expert nurses who have been working in the SICU for several years. In SICUN, we have formalized the cognitive apprenticeship framework posited by Collins, Brown, and Newman [1]. The framework includes six methods for developing an optimal learning environment: modeling, coaching, fading, articulation, reflection, and exploration.

Modeling, coaching, and fading represent an interconnected view of instruction and assessment. From an instructional perspective, models of cognitive competency can be provided as examples for new learners. Such modeling does not have to be intrusive. Students can problem solve independently for as long as they wish. When they want assistance they can ask for an alternative model of how an expert might solve the problem. Expert models are not directive in the sense that all students must follow an expert path. Rather, the models are provided as reflection opportunities where students can self-assess their solution steps with a more sophisticated peer. Norman stated that reflective thought requires deliberation, rather than an automatic reflex that is associated with experiential thinking [8]. Norman states that computers support reflective thinking when they enable users to compute new knowledge by adding new representations, modifying old ones, and comparing them. We will make the argument that SICUN can do just that in that it provides cognitive tools as reflection tools that amplify, extend, and reorganize human mental powers to help learners construct their own realities [9].

Instruction may consist of modeling the knowledge and skills that learners should learn. In this manner, instructors can make the goals of instruction clear so that learners will understand the types of knowledge that they will be assessed on. When difficulties are encountered in acquiring new knowledge, the computer can coach students with feedback that is designed to meet their level of skill. When the learner indicates that they can perform without difficulty, then coaching is faded or removed if it is no longer needed. Appropriate coaching and fading depends on an accurate assessment of individual performance in the context of a problem. Such assessments will drive the type and level of assistance generated. Fading of such assistance occurs when learners demonstrate that they can solve problems without assistance.

How are decisions reached regarding what skills to model in a particular domain? The content knowledge of the domain must be identified through cognitive task analysis methods that illustrate what competency means in a particular field. By studying different levels of expertise in particular problem solving contexts, a broad representation of the cognitive competencies can be identified. Expert strategies can then be modeled to the learner, and novices can receive coaching or feedback when they do not understand what they have observed. 
Such feedback can be adapted to an individual's needs and removed when no longer needed.

The other instructional methods supported by the cognitive apprenticeship framework are articulation, reflection, and exploration. Learners must be able to articulate their understanding, either through their performance (i.e., through menu selections), through oral explanations of their problem solving activities, or through written reports of their activities. Multiple mechanisms for articulation should be in place to accommodate individual differences in preferences for expressing their understanding. Instruction should also include opportunities for reflection on one's thinking. Computer traces of a learners' actions can be stored and replayed for learners, allowing them to reflect on their own problem solving skills. Finally, learners should be able to explore and test out new strategies. Instruction should be designed to promote such explorations through providing multiple methods for solving problems. In the section below a short review of the expertise literature is provided in an attempt to identify the types of skills that differentiate the proficient from less proficient in complex ill-structured problem solving domains.

\section{MODELING EXPERTISE}

Research results on expertise have accumulated as psychologists and cognitive scientists have investigated the underlying properties of human information processing and problem solving in various domains (for an extensive review see [10-12]). Studies employing the novice-expert paradigm to study clinical decision making indicate that, as compared with "less expert" medical personnel, experts, 1) have superior memory skills in recognizing patterns in their domain of expertise, 2) reason in a forward (data driven) manner (tend to solve the problem from the given information to a diagnostic hypothesis), 3) spend proportionally more time building a basic representation of the problem situation before searching for a solution, and 4) are opportunistic in terms of tuning their representation schemata when provided with additional medical information (having automatized many lower-level cognitive processes and being more capable of considering additional evidence that confirms or refutes their current hypotheses). These generalizations have been the source of much debate since evidence from numerous studies is contradictory. Still, the assumption underlying this paradigm is that the progression in clinical decision making from novice (e.g., undergraduate medical student) to expert (e.g., staff surgeon with 20 years of experience) is characterized by quantitative (e.g., enhanced recall of clinical cues) and qualitative (e.g., interconnected medical knowledge base and forward reasoning) differences [13]. These differences can be used to provide an understanding of cognitive development in the area of medical expertise and therefore have numerous pedagogical implications. 
However, the application findings from expert-novice studies have only indirectly contributed to models of learning and has been under-utilized in the design of innovative models of learning and assessment. Glaser, Lesgold, and Lajoie summarize much of the expert/novice literature on expertise in an attempt to guide principles for new measures of cognitive achievement that are driven by what we already know about skill acquisition [14]. These principles for examining dimensions of proficiency can be operationalized in innovative computer-based learning environments to promote the dynamic assessment of learning [15]. However, only a handful of systems for medical training have incorporated theory and methods from cognitive theories of effective learning and from research on the development of expertise [16]. Recent investigations of expertise are moving toward expanding our understanding of the conditions of learning and skill acquisition: 1) through the use of longitudinal studies focusing on the acquisition of expertise in individuals and effects of practice on changes in knowledge, component skills, and improvements in performance [17]; and, 2) by extending laboratory tasks to complex real-world domains [18-20].

\section{COMPLEX DECISION MAKING IN REAL WORLD DOMAINS}

The shift from laboratory tasks to complex real-world domains has acquired a shift in both the theoretical perspective and methodological approaches for studying expertise. The transition from controlled laboratory experiments to naturalistic environments requires the development of methodological and analytical methods to characterize the complex dynamics of real world cognition and the knowledge that is used to solve such problems. This also requires an expansion of the traditional IPT theory to account for cognition involving either individual or multiple agents performing real-world tasks in social contexts [21]. Recently, advocates of situated cognition have criticized the traditional IPT of cognition for its apparent inability to account for the flexibility and variability of human performance in real settings [22]. However, in this article we argue for an extension of the traditional IPT of cognition. Such an extension would necessitate to: 1) account for individual as well as group cognitive processes, 2) consider the dominant role of planning (including the coordination of multiple goals), ill-structuredness of complex real-world tasks, use of satisficing [56] methods, and situational assessment, 3) emphasize the mediating role of internal cognitive processing and situational factors/ assessment and their interaction in facilitating contextualized performance, and 4) adopt and develop research strategies for characterizing human performance on a range of cognitive activities from dynamic work activities to technological environments. 
There has been a proliferation of investigations of cognition in dynamic realworld environments (for an extensive review refer to [18, 20]). The range of domains studied include commercial and military aviation, structural and wildland firefighting, command and control operations, anesthesiology, nuclear power plant operations, software design, off-shore drilling management, corporate planning, jury deliberations, and highway design. This emerging area of research integrates conventional process-tracing methods (e.g., protocol analysis, cognitive task analysis) with innovative methods designed to investigate cognition and performance in real-world settings. Decision making research in such settings differs immensely from typical decision making research.

Complex decision making in real world domains has the following characteristics: 1) ill-structured problems, 2) incomplete, ambiguous, and changing information, 3) shifting, ill-defined, and competing goals, 4) decisions occur in multiple event-feedback loops, 5) time constraints, 6) stakes are high, 7) multiple participants contribute to the decision making process(es), and 8) the decision maker must balance personal choice with organizational norms and goals [23].

Training guidelines have been delineated for each of the above mentioned task characteristics [24]. The guidelines are based on task features and their corresponding desirable training procedures. For ill-structured problems, trainees should be provided with many trials in problem recognition and exposed to a wide variety of problem types. In dealing with action/feedback loops, trainees should be trained on multiple task components in a meaningful task context. In tasks involving heavy workload, training environments should provide "scaffolding" to reduce workload in early stages of training, and model and train experts' strategies for minimizing workload. To deal with the time stress and high stakes of particular settings, training should foster automaticity of task components, provide some training under stress, provide practice and feedback on making speed/accuracy tradeoffs, and train with simulated stressful conditions. In dealing with multiple players, training should emphasize performance monitoring, agenda setting, communication skills, and provide feedback on teamwork behaviors. Lastly, organizational goals and norms should involve organization members in the design and delivery of training, and determine how organizational "standards" may differentially affect performance. These training guidelines have served as the basis for the design of numerous models of naturalistic decision making. Klein has integrated various aspects of these models and formulated the Recognition-Primed Decision (RPD) model of naturalistic decision making [25]. The model attempts to describe what people actually do under conditions of time pressure, ambiguous information, ill-defined goals, and changing conditions. The descriptive model focuses on 1) experienced agents working in complex, uncertain conditions and 2) situational awareness and problem solving as part of decision making. 


\section{Clinical Decision Making in Nursing}

Investigators focusing on the cognitive aspects of clinical decision making have demonstrated that the decision making of nurses is complex and highly variable [e.g., 26]. Nurses vary their cognitive approach to decision making according to the complexity of the situation [27]. Nurses' decision making is characterized by the acquisition and cognitive organization of vast numbers of patient-generated cues [28, 29]. In addition, the formation of one or more diagnostic hypotheses has been found to guide nurses' information gathering and ultimate decision making $[28,30-32]$.

Researchers investigating other variables related to clinical decision making have studied the diagnostic reasoning of both nurses and nursing students [29]. The results have indicated that both use data-driven (i.e., forward reasoning) and cue-based data acquisition strategies to activate a hypothesis early in the diagnostic reasoning process. Similar to other research findings [28], novices tended to perceive a causal relationship between cues and states of health rather than a probabilistic one.

There is some controversy regarding the generalizability of the results on clinical diagnosing in nursing since numerous methodological inconsistencies have been identified. Furthermore, the results of most of these studies [e.g., 24] do not offer plausible explanations that account for the variability encountered in subjects' clinical decision making. More research is needed to identify the cognitive components that pertain to clinical decision making so that appropriate instruction can be created. Our approach to this problem is to conduct a cognitive task analysis of the domain prior to designing instruction such as that found in an ITS.

\section{LINKING THEORIES OF LEARNING TO THE DESIGN OF COMPUTER-BASED LEARNING ENVIRONMENTS}

Our paradigms are shifting with respect to how we think learners learn. In fact there currently are significant debates regarding where information processing procedures end and situated learning and constructivist philosophies begin [33-35]. Consequently, those designing computer-based leaming environments are struggling with how to best incorporate learning models in computer-based instruction [36]. One theme that has emerged is that computers can be considered as cognitive tools that aid cognition through interactive technologies that expand the mind [9,36-40]. Cognitive tools or mindtools can be thought of as tools that help students during thinking, problem solving, or learning [37]. Jonassen and Reeves request that researchers using these themes back up their claims with a constructivist philosophy, where students are active participants in constructing new knowledge through their interactions with technology [9]. Cognitive tools 
can help learners organize, restructure, and represent what they know. Derry and Lajoie have suggested that designing systems with cognitive tools could serve as a bridge between a strict student modeling camp, i.e., an intelligent tutoring camp that models student learning and provides assistance when needed, and a camp that does not use the computer to assess and model for the learner but serves as a platform for learning [36]. A middle camp perspective allows opportunities for dynamic assessment by the computer as well as freedom (albeit somewhat restricted) for students to pursue their own problem solving solutions, requesting assistance when needed. In the remainder of the article, we describe the real world domain we observed, the cognitive task analyses, and the SICUN system we designed as a result of our analyses.

\section{THE DOMAIN}

For the last several years we have worked with medical personnel at a major Montreal hospital to establish the appropriate knowledge base for the SICUN. The medical personnel include surgeons, surgical residents, surgical nurses, and respiratory technologists. The main objective for medical personnel working in the SICU is to intervene with patients that require Advanced Trauma Life Support ${ }^{\circledR}$ as a result of severe and personal injury. Personnel who work in this unit must be able to monitor patient responses using high-technology medical equipment. The SICU environment is a high information flow environment that involves monitoring: 1) the patients' motor movements and "vocalizations," 2) persistent ringing of alarms and signals emanating from complex medical equipment, and 3) patient data that comes from multiple sources. Clinical decision making in the SICU involves attending to multiple types of information in a dynamic setting.

Decision making in real-world domains such as the SICU often involves making complex decisions in situations where information is incomplete, ambiguous, and uncertain. Furthermore, problems encountered in such domains are often ill-structured and subject to a number of real-world constraints. Under such conditions of complexity and uncertainty, medical personnel may have to make rapid decisions by utilizing simplified decision making strategies.

Our research specifically targeted surgical nurses in an SICU in an effort to identify the cognitive components of clinical decision making in the SICU as it relates to patient assessment. A cognitive task analysis was performed in an effort to establish the skills that should be modeled in a computerized tutor for clinical decision making. Training medical personnel in clinical decision making using a computerized tutor is a major challenge since the nurses' role in decision making involves the careful selection of relevant patient information from an abundance of irrelevant data. Furthermore, the ITS approach advocated in this study stresses the development of curriculum issues which are aimed at standardizing medical 
training in a dynamic environment where becoming an "expert" usually involves many years of formal training.

\section{COGNITIVE TASK ANALYSIS AND ITS DEVELOPMENT}

ITS development involves the application of artificial intelligence (AI) techniques such as knowledge representation and inferencing to computer-based education and training. An ITS is an adaptive computer-based instructional system comprised of four modules: 1) an expert module, 2) a student model module, 3) a tutor module, and 4) a student-machine interface (for an extensive review refer to $[41,42])$. The rationale for building such complex instructional environments is based on the assumption that the learner's cognitive processes can be modeled, traced, and corrected in the context of problem solving [36, 43]. Further, the instructional approach is based on the outcomes of cognitive task analyses.

The purpose of a cognitive task is to assess the quantitative and qualitative differences in people's descriptions, concepts, principles, and procedures when engaged in performing a specific task [44-48]. Cognitive task analysis for tutor development is a multi-stage process, where researchers must first understand the most difficult aspects of the domain in question, and then construct tasks that will differentiate between skilled and less skilled performance in that domain. Means and Gott summarize these stages as identifying:

1. Tasks constituting the target performance for the job: What tasks represent the important and difficult portions of the job?

2. Skills involved in performing the target tasks: How are the problems solved when performed well? What kinds of procedural skills and system knowledge are involved?

3. Heuristics and mental models used by experts: Are there general strategies employed by experts across different types of problems? What are the nature and content of problem-solving plans used by experts? What mental models guide expert problem-solving, and in what manner?

4. Difficulties novices encounter: What do novices do when given these problems? What knowledge, skills, and strategies do they lack? Do they show consistent misconceptions? Are novices able to employ procedures in a variety of circumstances?

5. Where to concentrate teaching: Which of the skills, knowledge and strategies employed by experts are generally acquired through training? Where are the trouble spots that are either missed by current instruction or resistant to instruction? [49, pp. 38-39].

The cognitive task analysis of the SICU followed the same stages. Three head nurses from an SICU unit were interviewed to target the most difficult aspects of their job performance. Difficulty was described in terms of complexity of the 
patient case, as well as the level of a nurses' prior knowledge. An expert nurse in the SICU should be able to handle the most hemodynamically unstable patients, those who have compounded problems such as an organ or respiratory failure. Hemodynamically unstable patients are detected through a systematic assessment of a patient. The nurse monitors blood pressure, pulse, respiratory rate, pulmonary pressure, arterial pressures, and other hemodynamic parameters. If any of these parameters become unstable the patient may have a number of problems and a nurse will have to attend to all of these parameters in tracking the problem. Patient difficulty could become compounded by many types of disease situations such as ARDS (Adult Respiratory Distress Syndrome), where distress may start in the respiratory system and cause an imbalance in another system. As patient instability increases so do the medical procedures and the patients' needs. As severity increases so does the patient's instability. In handling this type of case the nurse would need sophisticated knowledge of the types of drugs involved in treating the case and the types of drug interactions or side effects. In addition, an expert nurse needs sophisticated background knowledge of the physiology of the disease process.

The transition in types of patient cases assigned to novice through expert is determined by amount of complexity, from single component problems to multicomponent problems. For instance, a novice may be assigned an overnight patient, stable patients, or patients that have one identifiable problem such as pancreatic transplant; an intermediate nurse may deal with more than one problem involving patient instability; and an expert nurse deals with multiple problems involving instability.

\section{WHAT DISTINGUISHES A SKILLED NURSE FROM A LESS SKILLED NURSE IN THE SICU?}

An expert is able to quickly and efficiently assess the situation in order to make a decision as to what to do next: 1) get the physician or 2) further monitor the patient's condition. Part of this ability involves predicting the consequences of a treatment regiment. In effect, all nurses have to attend to multiple sources of information but experts attend to more information of a greater complexity. Part of attending to multiple sources of information involves monitoring the medical devices attached to the patient, monitoring the information recorded on the flow sheet records that refer to the patients' changes in stability, as well as looking at the patient and noticing things that the numbers on the chart do not indicate (i.e., level of consciousness, color of skin, breathing).

Extensive interviews were conducted with nurses to discuss the scope of the curriculum for the types of problems and learning objectives that need to be addressed in the SICU. The researchers wanted to make sure that the types of problems addressed in the ITS would entail the most difficult parts of the job 
that a nurse would have to encounter. Following the interviews, the researchers worked intensively with expert nurses to establish problems in the SICU that would be useful for differentiating between expert and novice nurses. Once these problems were designed, structured interviews were conducted with personnel with varying levels of expertise.

\section{METHOD}

\section{Subjects}

Three SICU senior nurses at a large metropolitan university teaching hospital participated in the study. These nurses had between five to ten years of experience as SICU nurses and were encouraged to participate in this study by the SICU chief of staff. The small sample size is an accurate reflection of the domain, since SICU's are generally small in size. This causes difficulty in pulling subjects away from their work environment for research studies.

\section{Materials}

A complex trauma problem scenario of a male patient involved in a motor vehicle accident was developed by the head SICU nurse in conjunction with the research team. The written case description was used to elicit the cognitive components underlying the clinical decision making process used by the nurses. The case involves a patient who was hemodynamically unstable and suffering from hypovolemia. Hypovolemia is a critical illness involving fluid imbalances [50, 51]. Moreover, hypovolemia is a complex medical problem involving the circulatory, urinary, respiratory, and neurological systems [52, 53]. The case description:

Your patient is a twenty-seven-year-old male involved in a motor vehicle accident. He has suffered severe chest and facial injuries. These include a flail chest, a right hemopneumothorax, a right pulmonary contusion, and a right orbital fracture. Upon admission to the SICU (surgical intensive care unit) the patient had a right chest tube, two large bore peripheral IVs, and a nasogastric tube. He was intubated orally and mechanically ventilated. During the first twelve hours in SICU the patient required several boluses of ringer's lactate to maintain his systolic BP above $90 \mathrm{mmhg}$ and his urine output above $40 \mathrm{cc} / \mathrm{hr}$. He also received four units of packed red blood cells. During the past eight hours your patient's blood gases have deteriorated. His recent CXR shows a new onset of diffuse infiltrates. A Swanganz catheter has just been inserted. Now your patient's w/o (urinary output) has dropped to $20 \mathrm{cc} / \mathrm{hr}$. Please investigate and intervene. 


\section{Procedure}

Subjects were presented with a trauma patient case scenario and asked to think aloud while they solved the problem. The PARI methodology [see 45, 47] was used to elicit the think alouds. The PARI method is a semi-structured interviewing technique where the subjects are given a problem to solve and are asked a standard set of questions until they solve the problem. PARI refers to Prerequisite knowledge, Action, Result, Interpretation. For every action that a subject would like to conduct in the context of a problem, the interviewer asks the subject why they would do this action (what is the prerequisite for doing this action). Once an action is taken, the interviewer must state the result of the action, and the subject must state their interpretation of the result of this action. For example, when a nurse is asked to complete an assessment of the patient, a small sample of the PARI interview could be as follows:

Experimenter: Which body system would you start with?

Expert: Neurology system

Experimenter: WHY? (PREREQUISITE KNOWLEDGE)

Expert: I want to see if the patient is conscious.

Experimenter: What would you do first?

Expert: I would use my flashlight to examine reaction of the pupils (ACTION)

Experimenter: The pupils both react equally to the light stimulus by contracting. (RESULT) What does this result imply or mean: How do you interpret this?

Expert: There's no brain damage (it just means an important component of the CNS is functioning) (INTERPRETATION) . . .

Audio data was collected for each subject throughout the experimental sessions as they diagnosed the complex trauma case scenario vis-a-vis the patient's drop in urinary output. The objective was to assess the qualitative differences in the nurses' descriptions, concepts, principles, and procedures when engaged in clinical decision making. Each subject was instructed to: 1) read the written case description, and 2) think aloud as she/he investigated and intervened given the fact that the patient's urinary output has dropped to $20 \mathrm{cc} / \mathrm{hr}$. The researcher occasionally intervened during the interview and asked the subject for elaboration and/or clarification.

\section{Data Analysis}

The verbal protocols were transcribed, parsed, and coded for key aspects of clinical decision making and problem solving. A scheme for coding the subjects' protocol included categories for coding the following components of clinical decision making: 1) hypothesis generation, 2) the planning of medical interventions, 3) the actions performed, 4) the results of evidence gathering, 
5) interpretation of the results, and 6) the overall solution paths. Initially, two doctoral students working independently parsed the transcripts and constructed decision trees which were then validated by each subject and the consulting SICU nurse. Subsequently, definitive decision trees were constructed using the software package Inspiration ${ }^{\mathrm{TM}}$ to represent the critical aspects of each subject's clinical decision making.

\section{RESULTS}

All nurses arrived at the same solution but the verbal protocols indicated enormous variability in their clinical decision making processes, including: 1) the initial assessment that led to the conception of a problem statement, 2) the order in which the evidence was gathered, 3) the planning of medical intervention(s), 4) the actions performed, 5) the results of evidence gathering, 6) the interpretation of the results, and 7) the overall solution paths.

The results are based on the segmented verbal protocols in terms of the frequencies of the following problem solving behaviors: plans, actions, results, and interpretation made by each nurse (see Figure 1). The following provides a range of frequencies for each of these problem-solving behaviors (range for the three nurses): 11 to 13 percent consisted of planning of medical interventions, 29 to 37 percent was performing medical actions, 32 to 40 percent were results of evidence gathering, and finally 17 to 21 percent consisted of interpretations of results found. Each individual sub-plan was set to investigate a body system and

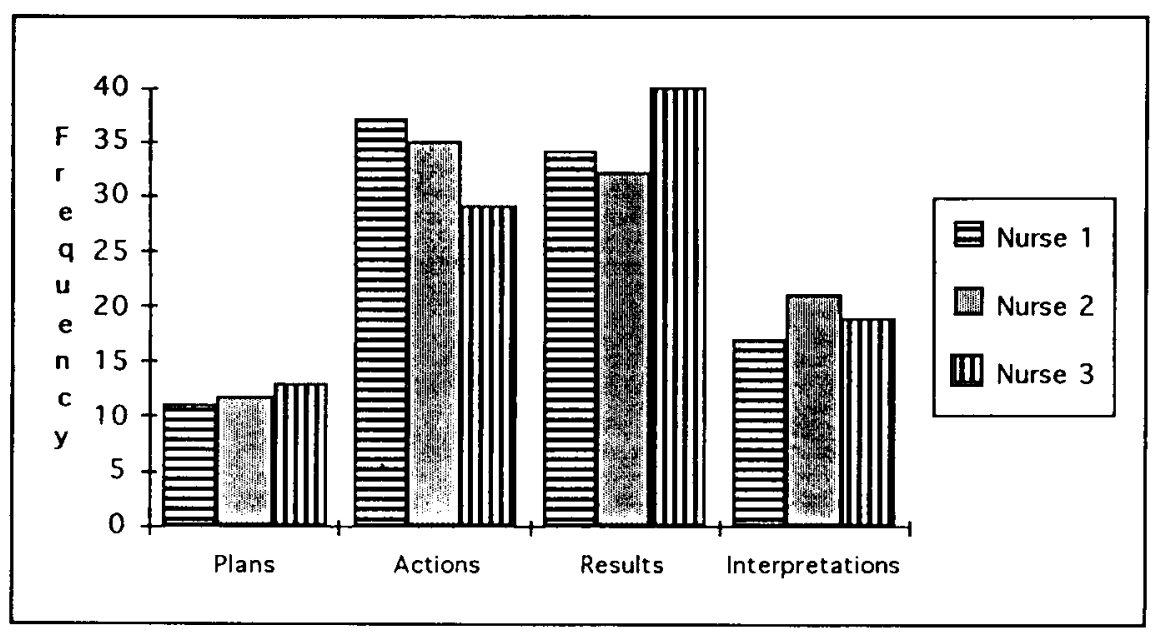

Figure 1. The frequency of plans, actions, results, and interpretations of three SICU nurses in solving the hypovolemia problem. 
thus aimed at verifying the hypothesized condition of hypovolemia. The results tend to fit a general clinical reasoning model which focuses on history-taking, physical examination, laboratory tests, $x$-rays, and specialized tests.

The modified effective problem space (EPS) of the three nurses' plans, subplans, actions, results, and interpretations supporting the correct diagnosis of hypovolemia is illustrated in Figure 2. As illustrated, solving the hypovolemia problem is associated with a total of eight plans, eight sub-plans, thirty-four actions, thirty-four results, and thirty-four interpretations. The shading in Figure 2 illustrates the aspects of the decision-making process that were common in the protocols of the three nurses. For example, they all created a plan to check the flow sheet to verify the patient's progression which indicated a low urinary output. This was then interpreted as the patient excreting too little urine. All of the nurses set plans to check the flow sheet, consult the multi-channel monitor, check the urinary system (including the quality of the urinary output), and check the patient's pulmonary function.

An idealized view of the medical decision-making process would involve: 1) settings plans (check urinary system), 2) creating sub-plans (check quality of urine output), 3) performing actions (check amount), and 4) interpreting the results (drop occurred in the amount). Such a systematic approach to clinical decision-making was not observed. Instead, enormous variability in the nurses' clinical decision-making was found (as indicated from the verbal protocols). The nurses' did not systematically create plans (or sub-plans), perform medical interventions (actions), retrieve the results of their interventions and readings from the medical devices, or interpret their findings.

For example, in brief, one nurse noted certain patient factors (drop in the patient's urinary output and a decrease in blood pressure) and proceeded to state that the patient had a problem associated with low volume. She proposed the hypothesis of hypovolemia and proceeded to gather evidence that would confirm this hypothesis. Her approach involved systematically examining the circulatory system, urinary system, respiratory system, integrity of the urinary drainage system, biochemistry results on the flow sheet, pulmonary functioning, blood/gas results, and hematology results. She was confident that all of the evidence gathered supported her hypothesis of hypovolemia. Lastly, she provided two possible solutions for counteracting the hypovolemia state: 1) to administer a unit of blood, or 2) to increase the ventilator's oxygen purity level from 40 to 50 percent.

A second nurse proceeded to verify the patient's respiratory system whereby she checked if the patient was well-intubated, ventilated, and the position of his E.T. tube. She then verified the patient's circulatory system by checking the multi-channeled monitor for the pulse rate (regular or irregular thus indicating arrhythmia), arterial line (e.g., blood pressure), and several other venous pressures (e.g., central venous pressure). Then she 1) checked the patient's abdomen with her hands for signs of guarding (i.e., hardness), 2) listened for presence or 
absence of bowel sounds, and 3) checked the urine output and realized the sharp drop in the patient's urine output. At this point in time, she postulated the hypothesis of hypovolemia. Subsequently, she continued her patient assessment by gathering further evidence confirming her hypothesis of hypovolemia. This was accomplished by checking the patient's neurological system by asking the patient to open his eyes, check pupil response to light, verbally command him to squeeze her hands, move his legs from side to side, and perform a sternal rub to verify his response to pain.

The third nurse decided to conduct a "head-to-toe" patient assessment. Before approaching the patient, she examined the situation: this is not an emergency case, the blood gases and blood pressure are within normal limits. She proceeded to examine the patient's neurological system by verifying his ability to respond to verbal commands. The neurological examination indicated to her that the patient was oriented. She then verified the respiratory system focusing on the performance of the patient's lungs by using a stethoscope (e.g., checking for adventitious sounds and wheezing) and then checked the E.T. tube (e.g., check tube placement and drainage). Next, she verified the patient's circulatory system to ensure that the perfusion rate was adequate by checking the skin (for swelling, edema, coloration, capillary refill, and temperature) and feeling his pulse. She then checked the patient's urinary output by visually inspecting the urinary bag and remarks that it is extremely low which led her to the hypothesis of hypovolemia. Subsequently, she verified his abdominal area by checking for bowel sounds (with the stethoscope) and abdominal "hardness." Finally, to confirm her hypothesis of hypovolemia she checked the patient's genitourinary system by checking the volume, color (clear or dark), and concentration of his urine.

\section{Summary}

Clinical decision making in nursing is complex and highly variable, as indicated by the SICU nurses' diagnostic reasoning in solving the hypovolemia case. This study: 1) presents an initial research study of dynamic clinical decision making in a real-world domain, 2) has implications for the initial design of a computerized tutor whose objective is to train medical personnel in clinical decision making, and 3) suggests the potential value of eliciting nurses' mental models as the basis for an instructional approach within the ITS.

\section{DESIGN OF THE SICUN TUTOR}

The design of SICUN is based on cognitive learning theories that emphasize the importance of situating learners in an actual context where they will apply their knowledge. The results of the cognitive task analyses suggest that clinical decision making is complex and multifaceted and as such the tutoring environment must provide for a multitude of problem solving sequences. Given the 


\begin{tabular}{|c|c|c|}
\hline PLAN & SUB-PLAN & ACTION \\
\hline \multirow[t]{7}{*}{$\begin{array}{l}\text { Check Flow Sheet } \\
\text { Consult Multi-Channel } \\
\text { Monitor }\end{array}$} & & $\begin{array}{l}\text { Check Patient's Progression } \\
\text { View E.C.G. }\end{array}$ \\
\hline & & $\begin{array}{l}\text { Check C.V.P. } \\
\text { Check P.W.P. } \\
\text { Check P.A.P (Systolic) }\end{array}$ \\
\hline & & $\begin{array}{l}\text { Check P.A.P (Mean) } \\
\text { Check P.A.P (Diastolic) }\end{array}$ \\
\hline & & Check E.C.G. \\
\hline & & Check Arterial Blood Pressure \\
\hline & Check Cardiac Output (C.O.) & $\begin{array}{l}\text { Inject 10cc of DW5 and watch \# } \\
\text { displayed on M.C.C. }\end{array}$ \\
\hline & Check Cardiac Index (C.I.) & Divide to C.O. by the body surface area \\
\hline \multirow[t]{7}{*}{ Check Urinary System } & & $\begin{array}{l}\text { Compare changes in urinary output over } \\
\text { time on flow sheet }\end{array}$ \\
\hline & $\begin{array}{l}\text { Check Quality of Urine Output } \\
\text { (by inspecting the bag) }\end{array}$ & Check amount \\
\hline & & Check color \\
\hline & & Check specific gravity \\
\hline & & Check urine lytes \\
\hline & $\begin{array}{l}\text { Check the Integrity of } \\
\text { Urinary Drainage System }\end{array}$ & $\begin{array}{l}\text { Check tubing and connections } \\
\text { Check bladder function }\end{array}$ \\
\hline & Check Bio-Chemistry Results & $\begin{array}{l}\text { Check B.U.N. (Blood Urea Nitrogen) } \\
\text { Check Createnine level }\end{array}$ \\
\hline \multirow{2}{*}{$\begin{array}{l}\text { Check Pulmonary } \\
\text { Function }\end{array}$} & & Osculate Patient's Chest \\
\hline & & $\begin{array}{l}\text { Check Chest Tube Drainage } \\
\text { Check Blood/Gas Results (pH) } \\
\text { Check Blood/Gas Results (PO2) } \\
\text { Check Blood/Gas Results (PCO2) } \\
\text { Check Blood/Gas Results (O2 Saturation } \\
\text { Check Patient's Respiratory Rate }\end{array}$ \\
\hline $\begin{array}{l}\text { Check Hematology } \\
\text { Results }\end{array}$ & & $\begin{array}{l}\text { Check Hematocrit level } \\
\text { Check Hemaglobin level }\end{array}$ \\
\hline $\begin{array}{l}\text { Check Patient's } \\
\text { Abdomen }\end{array}$ & & $\begin{array}{l}\text { Feel patient's abdomen by palpating } \\
\text { Listen for bowel sounds with stethoscope }\end{array}$ \\
\hline \multirow[t]{3}{*}{$\begin{array}{l}\text { Check Patient's } \\
\text { Neurological State }\end{array}$} & Check pupil area response & $\begin{array}{l}\text { Ask patient to open his eyes and shine } \\
\text { light in eyes }\end{array}$ \\
\hline & Check Tactile Response & $\begin{array}{l}\text { Ask patient to squeeze your hands and } \\
\text { move their legs }\end{array}$ \\
\hline & Check Pain Response & $\begin{array}{l}\text { Place hands on patient's sternum and } \\
\text { rub deeply }\end{array}$ \\
\hline
\end{tabular}

Figure 2. Effective problem space of three SICU nurses. 


\begin{tabular}{ll|}
\hline RESULT & INTERPRETATION \\
\hline Urinary Output is LOW & Too little Urine \\
B.P. has dropped from 120/80 to 100/80 &
\end{tabular}

\section{dropped from 8 to 6 \\ dropped from 12 to 8 \\ dropped (insert value) \\ dropped (insert value) \\ dropped (insert value)}

Change from sinus rhythm to Tachycardia

B.P. has dropped from $120 / 80$ to $100 / 80$

dropped from $4 \mathrm{~L} /$ minute to

3.2L/minute

dropped from $2.9 \mathrm{~L} / \mathrm{minute} / \mathrm{cm}-5$ to

$2.2 \mathrm{~L} / \mathrm{minute} / \mathrm{cm}-5$

urinary output dropped (70cc at 7a.m. to $20 c c$ at $8 a . m$.)

drop in amount

dark color

high (increased from 1.012 to 1.043 )

$\mathrm{Na}$ is high \& $\mathrm{K}$ is low

patent \& intact

not distended

increased over time from 10 to 15

normal (has remained the same)

\section{Clear bilateral air entry (no evidence of} coarse rowls)

No further blood loss \& fluctuation in the tube increased from 7.45 to 7.52

dropped from 95 to 80

dropped from 42 to 33

dropped from 95 to 89

increased respiratory rate from $12 \mathrm{bpm}$ to $18 \mathrm{bpm}$

elevated (insert \#)

dropped from 110 to 75

normal size and no guarding

absent

patient opens both eyes

patient obeys and moves both legs

decorticate and disseribrate responses
Pressure is related to volume

Urine dropping due to lack of volume to synthesize urine

All pressures have dropped due to lack of fluid in vascular space

Therefore, there is no urinary output

Solution, Perfusion to kidney

Patient is compensating for low volume by increasing the heart rate

Drop in blood pressure due to decreased volume

drop due to relative change in volume $\&$ provides evidence of low volume

drop due to relative change in volume $\&$ provides evidence of low volume

Volume depletion

Decreased amount of concentrated urine provides evidence of low volume

Decreased amount of concentrated urine provides evidence of low volume

Decreased amount of concentrated urine provides evidence of low volume

Refiects dehydration resulting from hypovolemia

Urinary drainage system is functional

No urinary retention

Should be normal (if HIGH is alludes to the hyp. of renal failure)

No evidence of pulmonary edema or fluid overload

Hypovolemia is not due to further bleeding in chest Patient is hyperventilating to compensate for Hypoxia

Patient is hyperventilating to compensate for Hypoxia Patient is hyperventilating to compensate for Hypoxia Patient is hyperventilating to compensate for Hypoxia Patient is hyperventilating to compensate for Hypoxia

May be leading to an indication that volume depletion is related to blood loss

Normal sign

Normal sign

Normal functioning

Normal functioning

Normal brain functioning 
ill-structured nature of the tasks there is no one best way to assess a patient, but there are ways in which novices can be taught to be more systematic in their clinical problem solving skills. Clinical decision making in the SICU involves attending to multiple sources of information in a dynamic setting.

The first consideration in designing the tutor was to provide an accurate representation of the SICU work environment. The interface must be authentic for nurses, surgical residents, and physicians. This environment includes a patient, a patient case history, patient records, medical devices required for patient care, a pharmacy from which to order and administer drugs, a medical tool kit (containing a stethoscope, etc.), and numerous tubing devices for everything from feeding to draining the patient (see Figure 3).

\section{Learner in the Context of a Problem}

The learner is a nurse starting her shift who performs a patient assessment on a twenty-seven-year-old male victim of a serious motor vehicle accident. The clock indicates when a learner begins or ends problem solving. When a patient is in critical care, the difference between life and death can often be measured by the

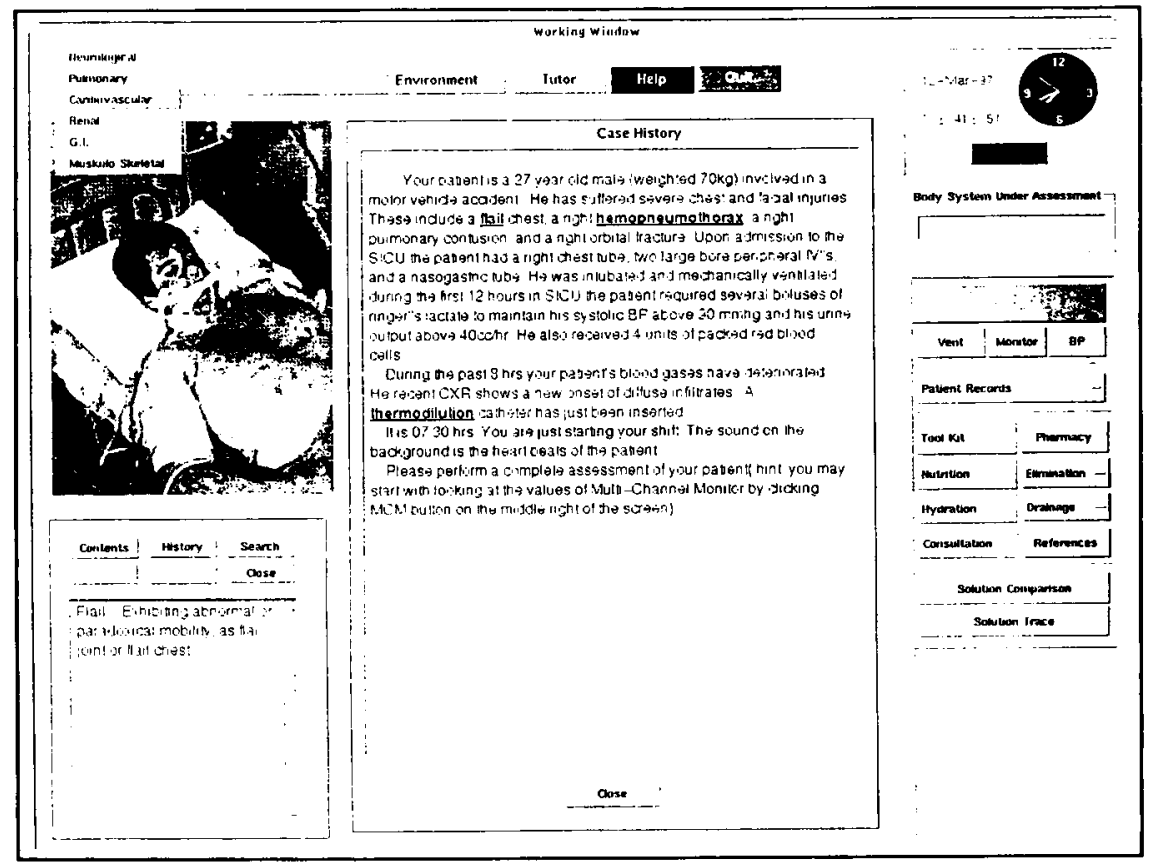

Figure 3. Initial case history presented in the SICU ITS. 
appropriate treatment performed within the appropriate time. Consequently, the SICU learning environment assesses the learners' problem solving time when considering their effectiveness.

Generally when performing a patient assessment a nurse will first read the case history and determine which body system to check. Much of the information presented in the case history is in hypertext, meaning that any terminology that is new to the learner, i.e., "flail chest," can be highlighted and further information about that term would be provided. In Figure 3 the cardiovascular system is selected. The systems menu represents an attempt to replicate the modified effective problem space depicted in Figure 2. The effective problem space decomposed nurses' decision making into plans and actions. The structure of the menu selections in the tutor allows nurses to indicate their plans by selecting a body system, such as cardiovascular, and follow up their plans by specific data collection actions (e.g., check pulmonary artery systolic pressure).

Once a system is selected the tutor prompts the nurse about what her goals are for assessing that system (see Figure 4). The tutor can then track the nurse's subsequent actions to see how they confirm or disconfirm their goals, in this case, checking perfusion status. Once the nurse moves on to another body system the

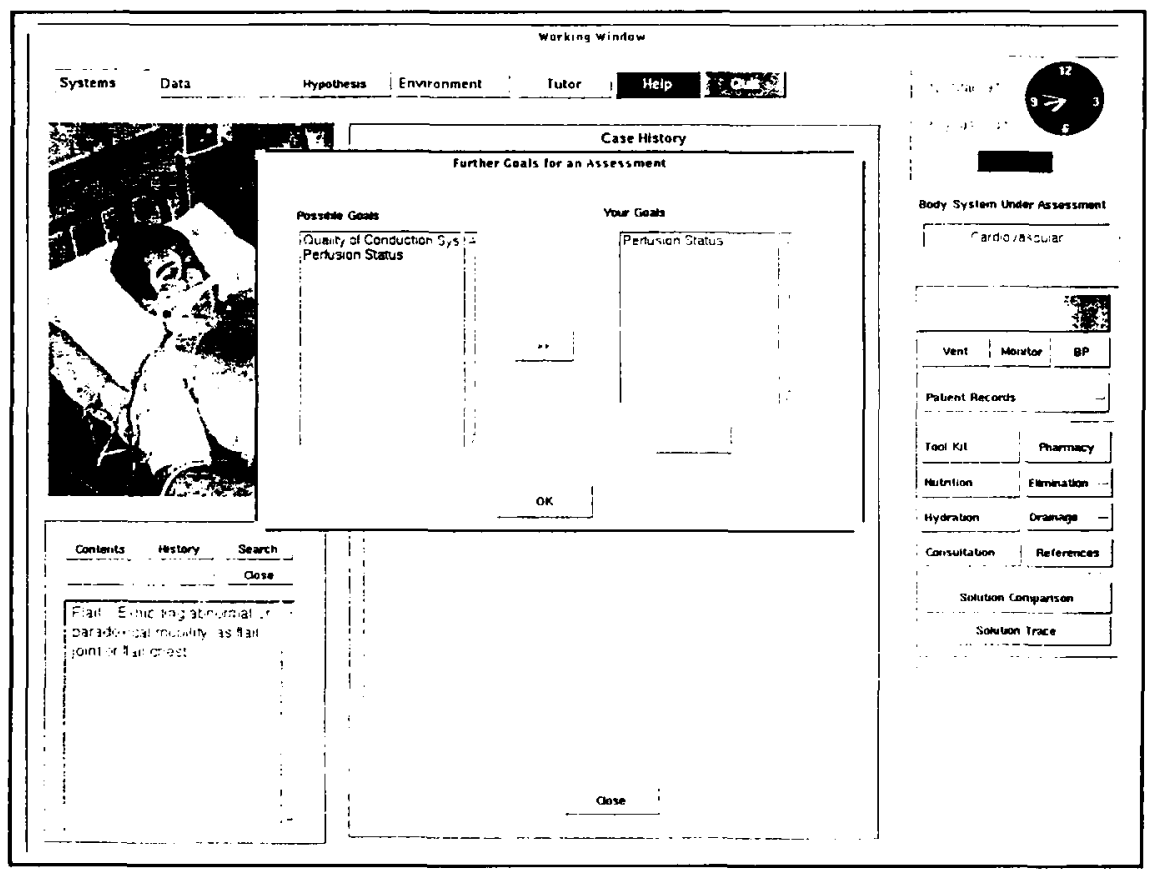

Figure 4. Explicit goal stating using the ITS dialog box. 
tutor will prompt them about the results of their assessment, in this case was perfusion status normal, altered, or severe. In Figure 5, different types of data can be collected from the patient based on selecting the cardiovascular system (check CVP, check PWP, etc.). The PAP systolic pressure has been selected, hence there is an image of the multi-channel monitor (MCM) displaying the patient data. Below the device image is a list of the patient variables that the MCM monitors. The PAP value is listed next to this list as being twenty-two. A nurse might request the patient's history over an eight-hour period on the PAP variable. At the bottom of the screen this history is displayed graphically as it would be in the patient flow sheet (a more complete set of patient records can be seen by selecting that button at the right of the screen image). Each record has dynamic and interactive functions associated with its many variables that are valuable for integrating time and events with the patient's evolving medical condition recorded at specific times. It demonstrates that the patients' PAP has been variable and the nurse interprets this data in the context of patient management. As mentioned earlier, the MCM and other medical devices can be selected from the palette located on the right hand side of the screen. Coupling this functionality

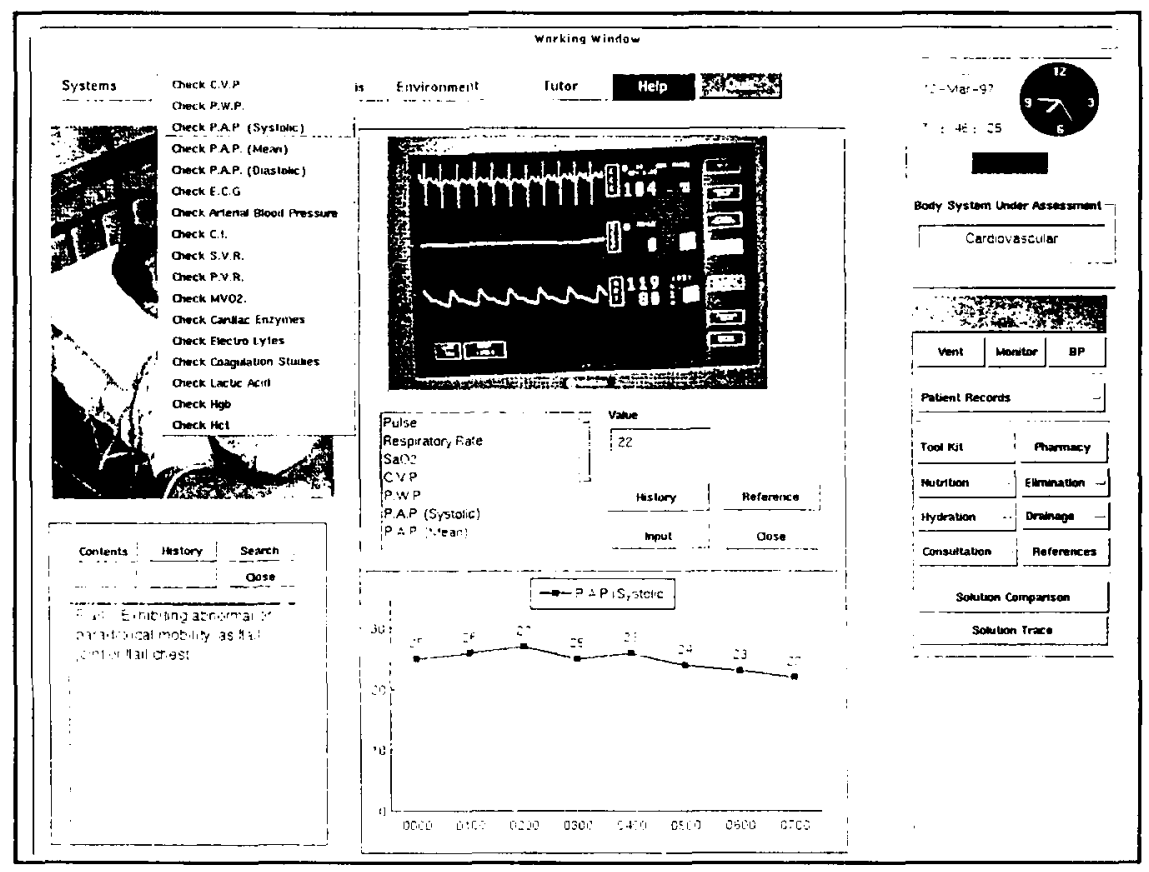

Figure 5. Cardiovascular parameters indicated on the Multichannel Monitor and Patient Records. 
with the systemic approach found under the systems button empowers the nurse practitioner to test her hypothesis and act on it in a simulated environment which replicates as much as possible the real world of the SICU.

The interface includes an extensive drug cabinet, accessible through the pharmacy button. A nurse may select a drug (e.g., Dopamine) based on the physicians' orders and then set up the Intravenous Baxter Pump ${ }^{\mathrm{TM}}$ as they would in the real world to infuse the drug into the patient. The nurse calculates the dilution rate based on the dosage and the patient's weight with an online calculator.

Nurses may formulate a hypothesis at any time. When the hypothesis button is selected in Figure 6, a list of possible diseases appears and the nurse can select up to three differential hypotheses and indicate their degree of confidence as to each diagnosis. In this case, hypovolemic shock is listed as most likely, followed by septic shock, and ARDS. These hypotheses can be re-weighted at any time, changed, or discarded.

Any time during problem solving the learner can ask to see their own solution trace by selecting the Solution Trace or Where am I button. They can also

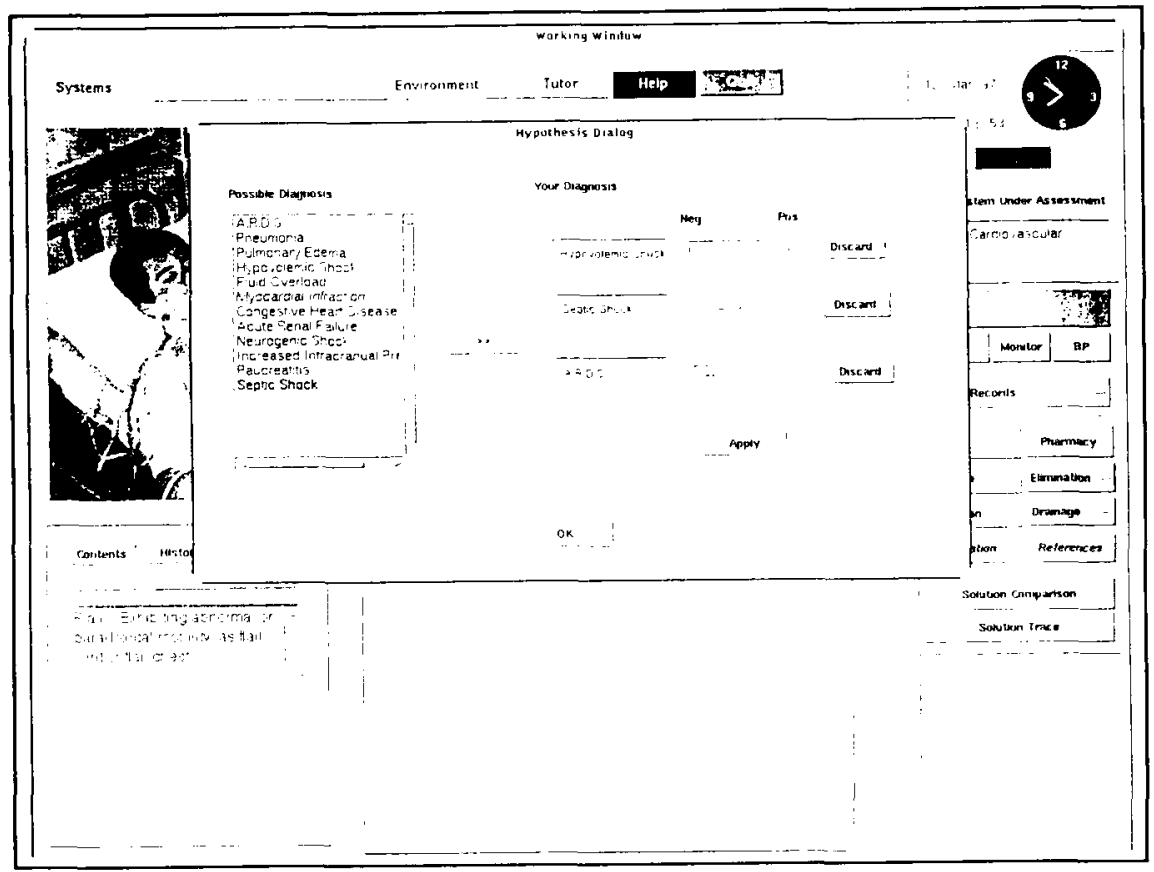

Figure 6. Listing differential hypotheses re. diseases and associated confidence (positive, negative) about one's hypotheses. 
compare their strategy to an experts' solution trace by selecting the solution comparison button (see Figure 7). This trace information provides a recapitulation of the specific plans and actions the nurse did in the context of the problem. These traces constrain the problem solving space by making the learner's actions visible.

\section{WHAT COGNITIVE TOOLS UNDERLIE THE DESIGN?}

The SICU tutoring environment is centered on the learning process as it occurs within situations. Hence, if learners encounter difficulty in the context of these situations then the appropriate information and assistance must be made available to them. Declarative or factual knowledge may be needed at various points throughout problem solving. Consequently, the tutor is designed with hypertext capabilities for specialized medical vocabulary and a reference library for information on any variables that are monitored by medical devices or appear in patient records. Much of what nurses do can be viewed as utilizing procedural knowledge, which is knowledge of how to perform some procedure or operation. For example, nurses must be able to load an intravenous pump with tubing and

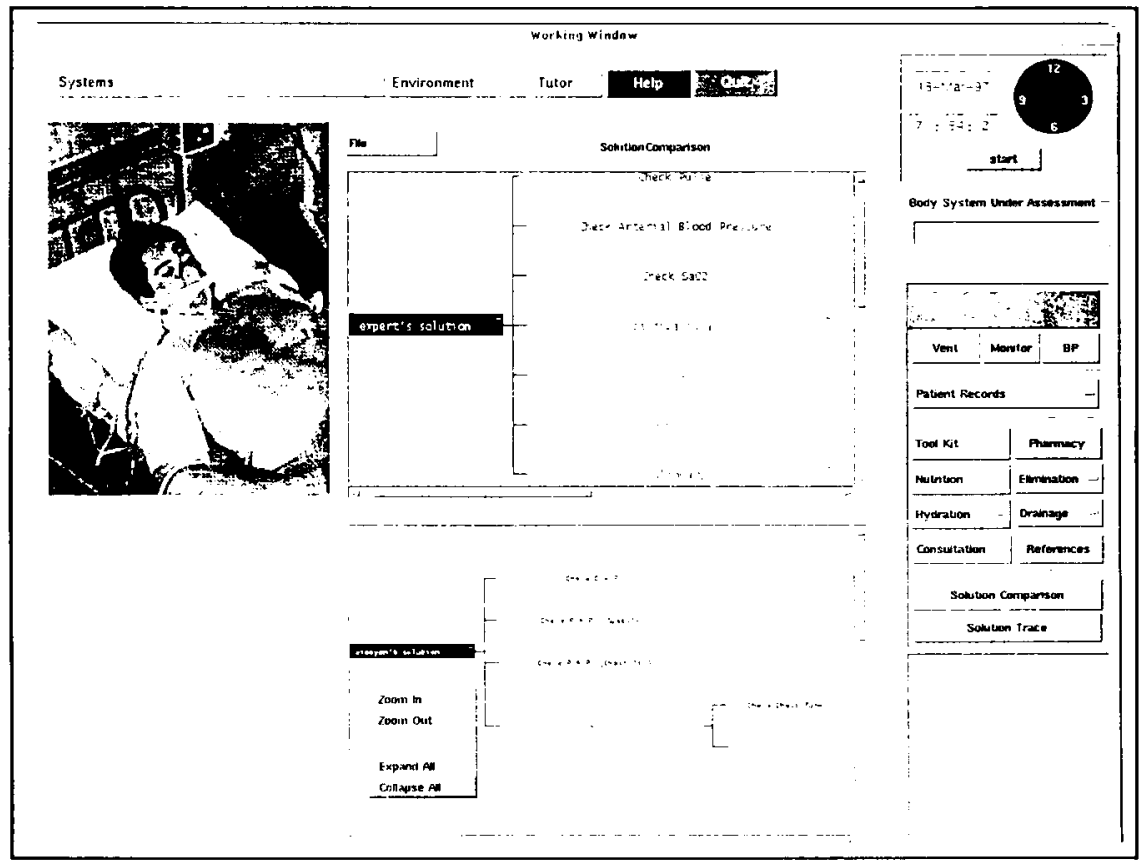

Figure 7. Solution comparison of learner and expert problem solving traces. 
medication and to set the rate of infusion appropriately for their patient. Consequently, the tutor demonstrates models of how to set up the Baxter pump, how to respond to alarms, and how to calculate the appropriate rates of infusion.

As described, the SICU is a high information flow environment and thus a learner may become cognitively overloaded and lose focus. The tutor reduces some of the attention burden by providing a memory aid. At any point throughout problem solving the nurse can ask to see her solution trace, which is a recap of the plans and actions she has taken prior to the request. This trace can help re-orient the problem solver, reminding them of what they have looked at. Another strain on the nurses' memory resources is their ability to keep track of all fifty-three patient variables that are recorded over time in the patient records. The graphical interface of the patient records has been designed to cluster these variables according to specific parameters, namely cardiovascular, biochemistry, hematology, ventilator, blood gases, and fluid balance. Furthermore, each variable is graphically plotted over time so a nurse can interpret radical changes in the patient's status. The graphical interface of the SICUN helps externalize the nurse's thoughts and actions as well as making an expert's plans and actions open for inspection. Technology has a place in making knowledge representations visible [9].

One of the pedagogical strategies used in the design of this tutor is making models of expertise available to learners. The goal in presenting such models is that learners can observe how decisions are made and compare their own strategies to those of experts. The tutor provides these opportunities at all times through the solution comparison button. Once learners observe these models they can reflect on their own strategies and self-assess their approaches to clinical decision making. The tutor provides explicit self-assessment prompts throughout problem solving. As demonstrated above, every time a nurse selects a body system to assess, she is prompted to stipulate her reasons or goals for the assessment. Subsequent to stipulating their goals nurses collect data for that phase of the patient assessment and move on to another body system. However, prior to starting a new assessment, the tutor prompts the user regarding their interpretations based on the data they collected. These prompts are designed to increase the nurses' metacognitive evaluation of their own reasoning to facilitate self-assessment.

A final feature of the graphical interface that needs elaboration is the hypothesis formation utility. Clinical decision making is prone to differential hypotheses rather than to single hypothesis testing. For this reason, there are three slots for entering possible hypotheses as to what diseases may be present. The hypothesis list is linked to a confidence meter. After a hypothesis is entered learners weight their level of certainty in their diagnosis. Nurses can discard, add, or re-weight their hypotheses at any time during their problem solving. This tool can provide a mechanism for assessing whether or not knowledge acquisition is correlated with increasing confidence. 


\section{HOW IS ASSESSMENT ADDRESSED?}

The tutor design facilitates the dynamic assessment of the learner in the context of problem solving. Assessment is dynamic in that it takes place while students are problem solving. The results of these assessments are used to generate appropriate levels of advice. Assessments are of learning processes rather than the learning outcomes. For instance, nurses are assessed on the types of decisions they make in the context of diagnosing the patient problems, how accurate the decisions are, how systematic they are in the actions and data they collect to confirm or disconfirm their hypotheses, how accurately they interpret patient data, how structured or fragmented their knowledge is as indicated by their solution strategies, how accurate their self assessments are, whether they use the metacognitive tools available to them, and whether they compare their solutions with those of experts. The tutoring environment is designed to help learners monitor their own progress and to compare their reasoning with that of others. The assumption behind this design feature is that when learners are made aware of what is expected of them, through models of expertise, it will be easier for them to meet those expectations, to assess their own progress, and compare their work with that of others. Furthermore, the relationship between knowledge acquisition with regard to diagnoses and confidence about diagnoses is assessed by SICUN. Finally, assessment occurs both within patient cases and throughout the curriculum of patient cases. In this manner, nurses' emerging knowledge can be examined over time.

\section{EVALUATION OF THE SICU TUTORING ENVIRONMENT}

The evaluation of the SICU tutoring environment is a multi-stage process. Currently, in the formative evaluation phase of the system, experts used SICUN and provided their input regarding the validity or authenticity of the environment, as well as information regarding the user interface (how easy it is to use the system). Pre- and post-tests (available upon request) were designed by a subject matter expert (a SICU nurse) in conjunction with the researchers to assess the types of knowledge (presented in the prototype) which are required to conduct patient assessments. These tests are designed to assess the types of knowledge learners have before and after interacting with the tutor. These assessments have been piloted tested. As a result SICUN is designed to dynamically assess the learners' knowledge within the environment.

The formative evaluation of the SICU tutoring environment was conducted with an expert nurse who had two years of experience in the SICU. The subject matter expert has been instrumental in all phases of this research. She has helped to develop the tutoring interface, coaching and demonstration scripts, preand post-tests, and the actual problem content. Given her familiarity with the field she was asked to introduce and demonstrate both the "demonstration" and 
"exploration" prototype modes of the SICU environment to an expert nurse (S). A pre-test was then administered to $S$, who then interacted with the system, and was subsequently given the post-test.

The expert nurse's $(S)$ partial problem solution trace while interacting with the exploratory SICUN is illustrated in Figure 8. This trace of information provides a recapitulation of the specific plans and actions that $S$ did in the context of solving the hypovolemia case. These traces have multiple on-line (problem-solving) uses, including: 1) to constrain the problem solving space by making the learner's actions visible to the system, 2) to provide the appropriate level of scaffolding and hinting depending on the pedagogical tutoring mode, and 3) to provide the user with the opportunity to compare their solution path to that of an expert (similar to example depicted in Figure 7).

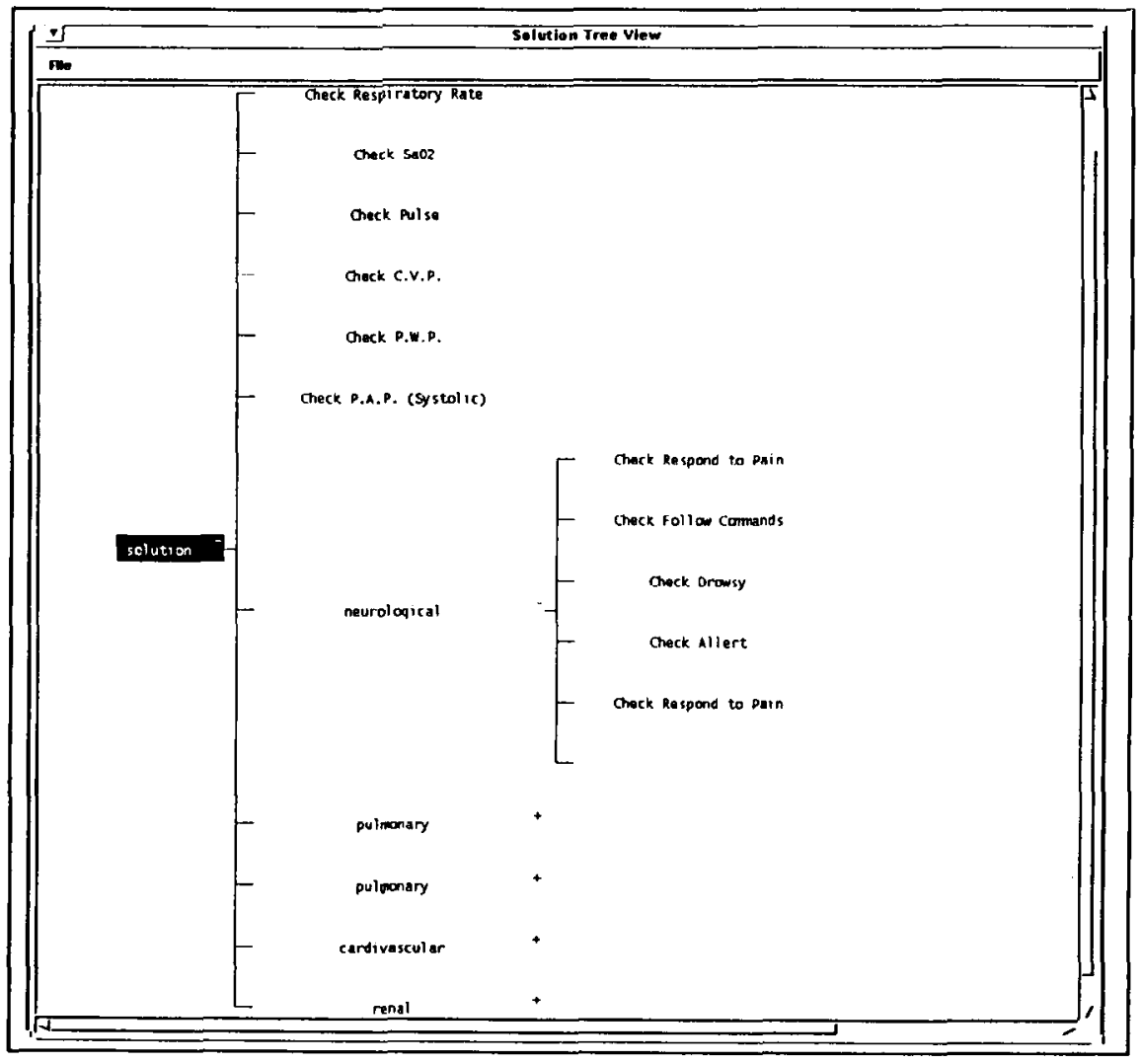

Figure 8. Partial expert nurse's (S) problem solving solution trace during the formative evaluation process. 
In assessing changes in $S$ from pre to post, it appears that $S$ explores the patient more thoroughly in the post-test since he pays more attention to a multitude of body systems. It is possible that SICUN had an influence on $S$ in making him more systematic in his patient assessments. The exploration mode may have helped $S$ to think out each system more completely and increased his awareness (of his thinking) during the assessment of the patient. S keys in on some specific data as opposed to getting a general view of the situation. It is possible that S's experience may have led him to a quicker solution, or that the problem scenario was too rich in information. The test results may have been quite different for a nurse with less experience. A novice nurse might be more thorough and planned in their actions but less specific in formulating hypotheses as to what may be a patient problem. For instance, $S$ stated he had difficulty in deciding which system to go to first. As stated, he is used to seeing all the relevant information at a glance on one big flowsheet and he has never been forced to describe his thinking process in a step by step format. Experts probably have a top level plan but do not usually articulate their plan. Instead it is quite possible that cues in the information flow chart evoke a patient schemata (of what could be wrong at that point in time), and consequently react to such information in a systematic manner.

In summary, our next steps will include: 1) testing both experts and novices with SICUN, using two pedagogical approaches (guided and discovery), and 2) refining the tutoring environment to meet the needs of the real world working environment.

\section{FUTURE DIRECTIONS}

Since research on rapid decision making in real-world domains is still quite rudimentary, plausible explanations which may account for the nurses' highly variable performance are presently unattainable. However, the complexity and variability revealed by the expert nurses' clinical decision-making can be partially explained within the framework of cognitive science literature in the areas of problem-solving and medical expertise. The lack of systematicity in the nurses clinical-decision making could be partially explained by the ill-structured nature of the complex medical trauma scenario and through the concept of bounded rationality [54]. This concept which simply states that since humans can rarely solve problems exactly, the optimizing strategy suggested by rational analysis [55] is seldom available. Instead of employing optimal problem-solving strategies, humans used "good-enough" strategies given the limitations of their computational capacity and speed, and the task and environmental complexity. The computational limits that are important in shaping behavior include the capacity of short-term memory, the presence and absence of external memory aids, the failure of humans to use strong problem-solving methods (due primarily to memory limitations), and the general absence of any optimization processes for selecting problem representations [56]. In addition, these results can be seen as an 
example of Holyoak's distinction between routine and adaptive expertise. Routine expertise involves solving familiar problems quickly and accurately [57]. However, adaptive expertise involves exhibiting flexibility in problem solving, being opportunistic by distinguishing between relevant and irrelevant incoming information, and applying metacognitive skills in regulating problem-solving behavior. Planning has recently been introduced as a key factor in skill acquisition and as a determining factor in successful complex problem-solving in illstructured tasks [58].

The results of this study illustrate the complexity of complex clinical decision making and the challenges associated with designing computerized tutors aimed at training medical personnel in ill-structured tasks. In dealing with the variability in problem solving, the SICU tutor interface is based on an isomorphic representation of the cognitive task analysis results. The results of the analyses allowed for the design of hierarchical menus that reflected the effective problem space of experts. Furthermore, the analyses served as a basis for the student model where the tutor could dynamically construct a problem-solving trace of the user that maps directly onto the effective problem space stored in the student model. This approach is more effective in terms of providing timely microadaptive and macroadaptive tutoring interventions while dealing with the variability in user's problem-solving approaches.

The next phase in this research is to evaluate the effectiveness of this tutor in terms of improving clinical reasoning in novices. Learner traces will be examined to look at strategy differences in clinical decision making, as well as the types of goals selected, and actions taken to complete a goal. Furthermore, different pedagogical strategies will be empirically tested to see which types of learners benefit from which strategies. A detailed examination of the effects of dynamic assessment and coaching will be examined. This type of research can provide detailed evidence regarding which types of computer tools are truly cognitive tools for learning and assessment.

\section{ACKNOWLEDGMENTS}

We gratefully acknowledge the other researchers and industrial partners: Claude Frasson, Jan Gecsei, Gilles Gauthier, Bernard Lefebvre, Marc Kaltenbach, Gilles Imbeau, Novasys and Virtual Prototype. We also acknowledge the solid work of the programmers, Wei Gu and Xiaoyan Zhao, Robert Bouchard a research assistant, and Julie Kinnon, our nursing expert, who has helped in all phases of this research.

\section{REFERENCES}

1. A. Collins, J. S. Brown, and S. E. Newman, Cognitive Apprenticeship: Teaching the Craft of Reading, Writing and Mathematics, in Knowing, Learning and Instruction: Essays in Honor of Robert Glaser, L. Resnick (ed.), Lawrence Erlbaum Associates, Hillsdale, New Jersey, pp. 453-494, 1989. 
2. J. S. Brown, A. Collins, and P. Duguid, Situated Cognition and the Culture of Learning, Educational Researcher, 18:1, pp. 32-42, 1989.

3. CTGV, Anchored Instruction and Its Relationship to Situated Cognition, Educational Researcher, 9:6, pp. 2-10, 1990.

4. E. W. Eisner, Forms of Understanding and the Future of Educational Research, Educational Researcher, 22:7, pp. 5-11, 1993.

5. J. G. Greeno, Perspectives on Thinking, American Psychologist, 44:2, pp. 134-141, 1989.

6. T. J. Shuell, Toward an Integrated Theory of Learning and Instruction, Educational Researcher, 28:4, pp. 291-311, 1993.

7. L. B. Resnick, Learning in School and Out, Educational Researcher, 12:9, pp. 13-20, 1987.

8. D. A. Norman, Some Observations on Mental Models, in Mental Models, D. Gentner and A. L. Stevens (eds.), Erlbaum, Hillsdale, New Jersey, pp. 7-14, 1983.

9. D. Jonassen and T. C. Reeves, Learning with Technology: Using Computers as Cognitive Tools, in Handbook of Research for Educational Communications and Technology, D. H. Jonassen (ed.), Macmillan, New York, pp. 693-719, 1996.

10. M. T. H. Chi, R. Glaser, and M. J. Farr, The Nature of Expertise, Lawrence Erlbaum Associates, Hillsdale, New Jersey, 1988.

11. K. A. Ericsson, The Road to Excellence: The Acquisition of Expert Performance in the Arts and Sciences, Sports and Games, Lawrence Erlbaum Associates, Mahwah, New Jersey, 1996.

12. K. A. Ericsson and J. Smith, Toward a General Theory of Expertise: Prospects and Limits, Cambridge University Press, Cambridge, Massachusetts, 1991.

13. V. Patel and G. Groen, The General and Specific Nature of Medical Expertise: A Critical Look, in Toward a General Theory of Expertise: Prospects and Limits, K. A. Ericsson and J. Smith (eds.), Cambridge University Press, Cambridge, Massachusetts, pp. 93-125, 1991.

14. R. Glaser, A. Lesgold, and S. P. Lajoie, Toward a Cognitive Theory for the Measurement of Achievement, in The Influence of Cognitive Psychology on Testing, Buros/Nebraska Symposium on Measurement, Vol. 3, R. Ronning, J. Glover, J. C. Conoley, and J. C. Witt (eds.), Lawrence Erlbaum Associates, Hillsdale, New Jersey, pp. 41-85, 1987.

15. S. P. Lajoie and A. Lesgold, Dynamic Assessment of Proficiency for Solving Procedural Knowledge Tasks, Educational Psychologist, 27:3, pp. 365-384, 1992.

16. S. I. Lillehaug and S. P. Lajoie, AI in Medical Education: Another Grand Challenge for Medical Informatics, Journal of Artificial Intelligence in Medicine, 12:3, pp. 1-29, 1998.

17. N. Charness, Expertise in Chess: The Balance between Knowledge and Search, in Toward a Genetic Theory of Expertise: Prospects and Limits, K. A. Ericsson and J. Smith (eds.), Cambridge University Press, New York, pp. 39-63, 1991.

18. G. A. Klein, J. Orasanu, R. Calderwood, and C. E. Zsambok, Decision Making in Action: Models and Methods, Ablex, Norwood, New Jersey, 1993.

19. V. L. Patel, D. R. Kaufman, and S. A. Magder, The Acquisition of Medical Expertise in Complex Dynamic Environments, in The Road to Excellence: The Acquisition of 
Expert Performance in the Arts and Sciences, Sports and Games, Erlbaum, Mahwah, New Jersey, 1996.

20. C. E. Zsambok and G. Klein, Naturalistic Decision Making, Erlbaum, Mahwah, New Jersey, 1997.

21. J. G. Greeno, The Situativity of Knowing, Learning, and Research, American Psychologist, 53:1, pp. 5-26, 1998.

22. W. J. Clancey, Situated Action: A Neuropsychological Interpretation Response to Vera and Simon, Cognitive Science, 17, pp. 87-116, 1993.

23. J. Orasanu and T. Connolly, The Reinvention of Decision Making, in Decision Making in Action: Models and Methods, G. A. Klein, J. Orasanu, R. Calderwood, and C. E. Zsambok (eds.), Ablex, Norwood, New Jersey, pp. 327-345, 1993.

24. B. Means, E. Salas, B. Crandall, and T. O. Jacobs, Training Decision Makers for the Real World, in Decision Making in Action: Models and Methods, G. Klein, J. Orasanu, R. Calderwood, and C. E. Zsambok (eds.), Ablex, Norwood, New Jersey, pp. 306-326, 1993.

25. G. Klein, Naturalistic Decision Making: Where Are We Going? in Naturalistic Decision Making, C. E. Zsambok and G. Klein (eds.), Erlbaum, Mahwah, New Jersey, pp. 383-398, 1997.

26. J. M. Jenkins, The Pattern of Personal Knowing in Nurse Clinical Decision Making, Journal of Nursing Education, 32:9, pp. 399-405, 1993.

27. S. A. Corcoran, Planning by Expert and Novice Nurses in Cases of Varying Complexity, Research in Nursing and Health, 9, pp. 155-162, 1986.

28. J. Itano, A Comparison of Clinical Judgment Process in Experienced Registered Nurses and Student Nurses, Journal of Nursing Education, 28, pp. 120-126, 1989.

29. C. Tanner, K. Padrick, U. Westfall, and D. Putzier, Reasoning Strategies of Nurses and Nursing Students, Nursing Research, 36, pp. 358-363, 1987.

30. S. A. Corcoran, Task Complexity and Nursing Expertise as Factors in Decision Making, Nursing Research, 35, pp. 107-112, 1986.

31. K. Padrick, C. Tanner, D. Putzier, and U. Westfall, Hypothesis Evaluation: A Component of Diagnostic Reasoning, in Classifying of Nursing Diagnosis: Proceedings of the Seventh National Conference, A. McLane (ed.), C. V. Mosby Company, St. Louis, Missouri, pp. 299-305, 1987.

32. D. Putzier, K. Padrick. U. Westfall, and C. Tanner, Diagnostic Reasoning in Critical Care Nursing, Heart and Lung, 14, pp. 430-437, 1985.

33. J. R. Anderson, L. M. Reder, and H. A. Simon, Situated Learning and Education, Educational Researcher, 25:4, pp. 5-11, 1996.

34. R. Donnoyer, Introduction: This Issue: Refocusing on Learning, Educational Researcher, 26:1, pp. 4-34, 1997.

35. R. E. Mayer, Learners as Information Processors: Legacies and Limitations of Educational Psychologies Second Metaphor, Educational Psychologist, 31:3/4, pp. 151-161, 1997.

36. S. J. Derry and S. P. Lajoie, A Middle Camp for (Un)intelligent Instructional Computing: An Introduction, in Computers as Cognitive Tools, S. P. Lajoie and S. J. Derry (eds.), Lawrence Erlbaum Associates, Hillsdale, New Jersey, pp. 1-11, 1993.

37. D. H. Jonassen, Computers in the Classroom: Mindtools for Critical Thinking, Prentice Hall, Columbus, Ohio, 1996. 
38. P. Kommers, D. H. Jonassen, and T. Mayes (eds.), Cognitive Tools for Learning, Springer, Berlin, 1992.

39. R. D. Pea, Beyond Amplification: Using the Computer to Reorganize Mental Functioning, Educational Psychologist, 20, pp. 167-182, 1985.

40. G. Salomon, D. N. Perkins, and T. Globerson, Partners in Cognition: Extending Human Intelligence with Intelligent Technologies, Educational Researcher, 20, pp. 10-16, 1991.

41. J. E. Greer and G. I. McCalla, Student Modelling: The Key to Individualized Knowledge-Based Instruction, NATO ASI Series, Vol. 125, Springer-Verlag, Berlin, 1994.

42. V. J. Shute and J. Psotka, Intelligent Tutoring Systems: Past, Present and Future, in Handbook of Research on Educational Communications and Technology, D. Jonassen (ed.), Scholastic Publications, New York, pp. 1-99, 1994.

43. S. P. Lajoie, Computer Environments as Cognitive Tools for Enhancing Learning, in Computers as Cognitive Tools, S. P. Lajoie and S. J. Derry (eds.), Lawrence Erlbaum Associates, Hillsdale, New Jersey, pp. 261-288, 1993.

44. A. Lesgold and S. Lajoie, Complex Problem Solving in Electronics, in Complex Problem Solving: Principles and Mechanisms, R. J. Sternberg and P. A. Frensch (eds.), Lawrence Erlbaum Associates, Hillsdale, New Jersey, pp. 287-316, 1991.

45. A. Lesgold, S. P. Lajoie, D. Logan, and G. M. Eggan, Cognitive Task Analysis Approaches to Testing, in Diagnostic Monitoring of Skill and Knowledge Acquisition, N. Frederiksen, R. Glaser, A. Lesgold, and M. Shafto (eds.), Lawrence Erlbaum Associates, Hillsdale, New Jersey, pp. 325-350, 1990.

46. A. Lesgold, S. Lajoie, R. Eastman, G. Eggan, D. Gitomer, R. Glaser, L. Greenberg, D. Logan, M. Malone, A. Weiner, R. Wolf, and L. Yengo, Cognitive Task Analysis to Enhance Technical Skills Training and Assessment, University of Pittsburgh, Learning Research and Development Center, Pittsburgh, 1986.

47. B. Means, Cognitive Task Analysis as a Basis for Instructional Design, in Cognitive Science Foundations of Instruction, M. Rabinowitz (ed.), Lawrence Erlbaum Associates, Hillsdale, New Jersey, pp. 97-118, 1993.

48. J. M. Ryder and R. E. Redding, Integrating Cognitive Task Analysis into Instructional Systems Development, Educational Technology Research \& Development, 41:2, pp. 75-96, 1993.

49. B. Means and S. P. Gott, Cognitive Task Analysis as a Basis for Tutor Development: Articulating Abstract Knowledge Representations, in Intelligent Tutoring Systems: Lessons Learned, J. Psotka, L. D. Massey, and S. A. Mutter (eds.), Lawrence Erlbaum Associates, Hillsdale, New Jersey, pp. 35-57, 1988.

50. B. C. Mims, Case Studies in Critical Care Nursing, Williams \& Wilkins, Baltimore, Maryland, 1990.

51. P. L. Swearingen and J. H. Keen, Manual of Critical Care: Applying Nursing Diagnoses to Adult Critical Illness (2nd Edition), Mosby Year Book, St. Louis, 1991.

52. S. M. Williams, Decision Making in Critical Care Nursing, B. C. Decker, Inc., Burlington, Ontario, 1992.

53. J. M. Wilkinson, Nursing Process in Action: A Critical Thinking Approach, AddisonWesley Nursing, Redwood City, California, 1992. 
54. H. A. Simon, The Sciences of the Artificial (2nd Edition), MIT Press, Cambridge, Massachusetts, 1978.

55. J. R. Anderson, Rules of the Mind, Lawrence Erlbaum Associates, Hillsdale, New Jersey, 1993.

56. H. A. Simon, Cognitive Architectures and Rational Analysis: Comment, in Architectures for Intelligence: The Twenty-Second Carnegie Mellon Symposium on Cognition, K. VanLehn (ed.), Lawrence Erlbaum Associates, Hillsdale, New Jersey, 1991.

57. K. J. Holyoak, Symbolic Connectionism: Toward Third-Generation Theories of Expertise, in Toward a General Theory of Expertise: Prospects and Limits, K. A. Ericsson and S. Smith (eds.), Cambridge University Press, Cambridge, Massachusetts, pp. 301-336, 1991.

58. K. VanLehn, Cognitive Skill Acquisition, Annual Review of Psychology, 47, pp. 513-539, 1996.

Direct reprint requests to:

Dr. Susanne P. Lajoie

McGill University

Department of Education \& Counselling Psychology

3700 McTavish Street

Montreal, Quebec

H3A 1 Y2 